Associated adverse outcomes and risk factors including intraoperative transesophageal echocardiography. J

Thorac Cardiovasc Surg 1995; 110: 517-22.

\section{Ventilation of a patient after esophageal intubation with a double- lumen tube}

To the Editor:

Sometimes, intubation with a double-lumen tube (DLT) is difficult. We present a patient who was ventilated after esophageal intubation with a DLT used like an esophageal-endotracheal combitube (EEC). A 66-yr-old female patient $(155 \mathrm{~cm}, 74 \mathrm{~kg}$, body mass index $=31$ ) was scheduled for thymectomy for gradeI myasthenia gravis. Her thyromental distance was less than $6 \mathrm{~cm}$ and difficult intubation was predicted. General anesthesia was induced with $70 \mathrm{mg}$ propofol and $0.1 \mathrm{mg}$ fentanyl, and maintained with $4 \%$ sevoflurane. She was intubated without using a muscle relaxant with a 35 Fr-Left-DLT (Sheridan, Tyco, Tokyo, Japan). Her Cormack's laryngoscopic view was grade 3. Although the DLT could not be advanced more

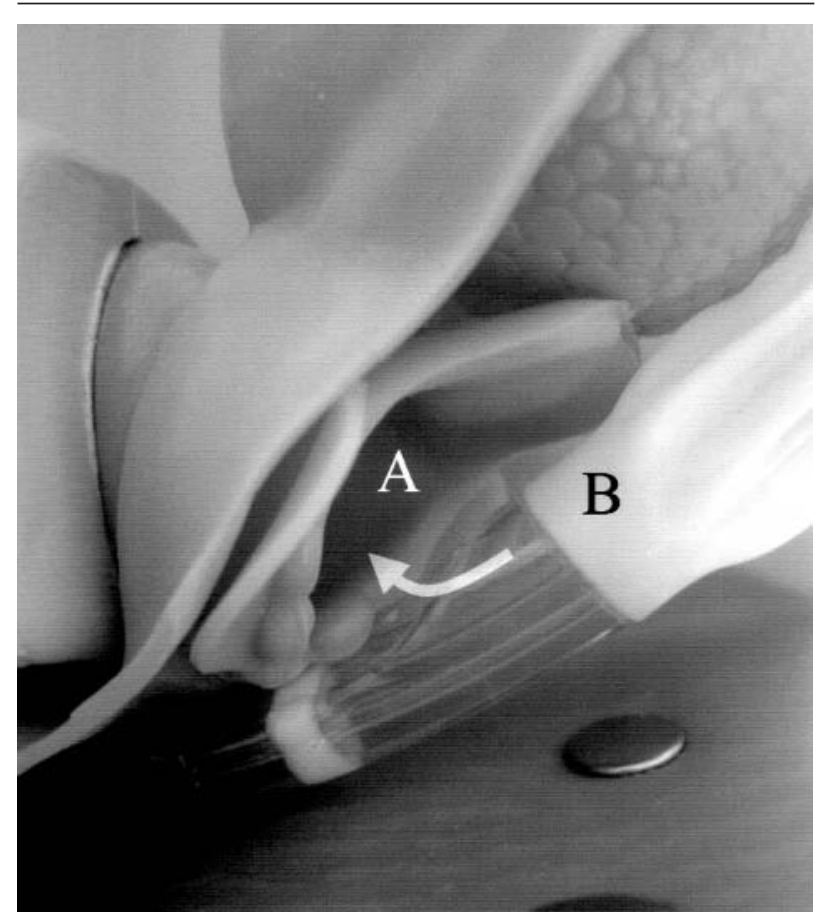

FIGURE Relationship between the vocal cords and the tracheal lumen of a double- lumen tube (DLT). The bronchial lumen is inserted in the esophagus. A, vocal cords; B, tracheal lumen of the DLT. than $21 \mathrm{~cm}$ from the patient's incisors, endotracheal intubation was assumed when spontaneous breathing re-appeared rapidly and capnography detected a normal $\mathrm{CO}_{2}$ waveform. However, the patient could not be ventilated mechanically. Subsequent fibroscopy via the bronchial lumen revealed esophageal intubation. The bronchial lumen compressed the posterior wall of the esophageal inlet, while the tracheal lumen opened just posterior to the arytenoid cartilages (Figure). In this case, the DLT performed as an EEC. Grmec reported that capnography was the most reliable method to confirm esophageal intubation, ${ }^{1}$ since the $\mathrm{CO}_{2}$ waveform generally decreases to zero, even if an initial pulse of swallowed $\mathrm{CO}_{2}$ is sensed. However, repeated airway evaluations should be performed to prevent a misdiagnosis such as occurred in our case, rather than depending on capnography alone.

Junichi Ogata MD

Toru Yokoyama MD

Kouichiro Minami MD PhD

Fukuoka, Japan

\section{Reference}

1 Grmec $S$. Comparison of three different methods to confirm tracheal tube placement in emergency intubation. Intensive Care Med 2002; 28: 701-4.

\section{Management choices for the difficult airway}

\section{To the Editor:}

We read with great concern the article by Jenkins et al. (October 2002). ${ }^{1}$ Their survey showed that in "Clinical Scenario number 3 - Laryngeal tumour with stridor for laryngectomy," 90\% of anesthesiologists would perform an awake intubation, $45 \%$ of which would use fibreoptic bronchoscope (FOB) and 38\% a lighted stylet.

This is very dangerous practice and could result in death of the patient. Best anesthetic practice is either 1) gas induction of anesthesia, maintaining spontaneous respiration, and when deep enough using a rigid laryngoscope (our preference is the tubular Lindholm scope as it opens up the posterior pharyngeal space and pushes tumour or edema out of the way). 2) If the patient is deemed to have an extremely compromised airway then an awake surgical tracheostomy is indicated. ${ }^{2}$

Awake FOB intubation is totally contraindicated in this scenario for the following reasons: 